\title{
ARCANGELI'S TYPE DISCREPANCY PRINCIPLES FOR A CLASS OF REGULARIZATION METHODS USING A MODIFIED PROJECTION SCHEME
}

\author{
M. T. NAIR AND M. P. RAJAN
}

Received 22 June 2001

Solodkiu (1998) applied the modified projection scheme of Pereverzev (1995) for obtaining error estimates for a class of regularization methods for solving ill-posed operator equations. But, no a posteriori procedure for choosing the regularization parameter is discussed. In this paper, we consider Arcangeli's type discrepancy principles for such a general class of regularization methods with modified projection scheme.

\section{Introduction}

Regularization methods are often employed for obtaining stable approximate solutions for ill-posed operator equations of the form

$$
T x=y,
$$

where $T: X \rightarrow X$ is a compact linear operator on a Hilbert space $X$. It is well known that if $R(T)$ is infinite dimensional, then the problem of solving the above equation is ill-posed, in the sense that the generalized solution $\hat{x}:=T^{\dagger} y$ does not depend continuously on the data $y$. Here, $T^{\dagger}$ is the generalized MoorePenrose inverse of $T$ defined on the dense subspace $D\left(T^{\dagger}\right):=R(T)+R(T)^{\perp}$ of $X$, and $R(T)$ denotes the range of the operator $T$. A typical example of such an ill-posed equation is the Fredholm integral equation of the first kind

$$
\int_{a}^{b} k(s, t) x(t)=y(s), \quad a \leq s \leq b
$$

with $X=L^{2}[a, b]$, and $k(\cdot, \cdot)$ a nondegenerate kernel belonging to $L^{2}([a, b] \times$ $[a, b])$. 
In a regularization method, corresponding to an inexact data $\tilde{y}$, one looks for a stable approximation $\tilde{x}$ of $\hat{x}$ such that $\|\hat{x}-\tilde{x}\|$ is "small" whenever the data error $\|y-\tilde{y}\|$ is "small." A well-studied class of regularization methods for such a purpose is characterized by a class of Borel functions $g_{\alpha}, \alpha>0$, defined on an interval $(0, b]$ where $b \geq\|T\|^{2}$. Corresponding to such functions $g_{\alpha}$, the regularized solutions are defined by

$$
x_{\alpha}:=g_{\alpha}\left(T^{*} T\right) T^{*} y, \quad \tilde{x}_{\alpha}:=g_{\alpha}\left(T^{*} T\right) T^{*} \tilde{y} .
$$

(Cf. [1].) In order to perform error analysis, we impose certain conditions on the functions $g_{\alpha}, \alpha>0$. Two primary assumptions are the following.

Assumption 1. There exists $v_{0}>0$ such that for every $v \in\left(0, v_{0}\right]$, there exists $c_{v}>0$ such that

$$
\sup _{0 \leq \lambda \leq b} \lambda^{\nu}\left|1-\lambda g_{\alpha}(\lambda)\right| \leq c_{\nu} \alpha^{\nu} \quad \forall \alpha>0
$$

Assumption 2. There exists $d>0$ such that

$$
\sup _{0 \leq \lambda \leq b} \lambda^{1 / 2}\left|g_{\alpha}(\lambda)\right| \leq d \alpha^{-1 / 2} \quad \forall \alpha>0
$$

These assumptions are general enough to include many regularization methods such as the ones given below.

For applying our discrepancy principle, we would like to impose two additional conditions.

Assumption 3. There exist $\alpha_{0}>0$ and $\kappa_{0}>0$ such that

$$
\left|1-\lambda g_{\alpha}(\lambda)\right| \geq \kappa_{0} \alpha^{\nu_{0}} \quad \forall \lambda \in[0, b], \forall \alpha \leq \alpha_{0}
$$

Assumption 4. The function $f(\alpha)=\alpha^{q}\left[1-\lambda g_{\alpha}(\lambda)\right], q>0$, as a function of $\alpha$, is continuous and differentiable and $f(\alpha)$ is an increasing function.

Now we list a few regularization methods which are special cases of the above procedure.

\section{Tikhonov regularization}

$$
\left(T^{*} T+\alpha I\right) x_{\alpha}=T^{*} y
$$

Here

$$
g_{\alpha}(\lambda)=\frac{1}{\lambda+\alpha} .
$$

Assumptions 1, 2, 3, and 4 hold with $\nu_{0}=1$, and $\kappa_{0}$ in Assumption 3 can be taken as greater than or equal to $1 /\left(\alpha_{0}+\|T\|^{2}\right)$. 
Generalized Tikhonov regularization

$$
\left(\left(T^{*} T\right)^{q+1}+\alpha^{q+1} I\right) x_{\alpha}=\left(T^{*} T\right)^{q} T^{*} y .
$$

Here

$$
g_{\alpha}(\lambda)=\frac{\lambda^{q}}{\lambda^{q}+\alpha^{q+1}} .
$$

Assumptions 1, 2, 3, and 4 hold with $v_{0}=q+1, q \geq-1 / 2$, and $\kappa_{0}$ in Assumption 3 can be taken greater than or equal to $1 /\left(\alpha_{0}^{q+1}+\|T\|^{2(q+1)}\right)$.

Iterated Tikhonov regularization. In this method, the $k$ th iterated approximation $x_{\alpha}^{(k)}$ is calculated from

$$
\left(T^{*} T+\alpha I\right) x_{\alpha}^{(i)}=\alpha x_{\alpha}^{(i-1)}+T^{*} y, \quad i=1, \ldots, k,
$$

with $x_{\alpha}^{(0)}=0$. Here, with

$$
g_{\alpha}(\lambda)=\frac{1}{\lambda}\left[1-\left(\frac{\alpha}{\alpha+\lambda}\right)^{k}\right] .
$$

Assumptions 1, 2, 3, and 4 hold with $v_{0}=k$ and the constant $\kappa_{0}$ in Assumption 3 can be taken as any number greater than or equal to $1 /\left(\alpha_{0}+\|T\|^{2}\right)^{k}$.

In order to obtain numerical approximations of $\tilde{x}_{\alpha}=g_{\alpha}\left(T^{*} T\right) T^{*} \tilde{y}$, one may have to replace $T$ by an approximation of it, say by $T_{n}$, where $\left(T_{n}\right)$ is a sequence of finite rank bounded operators which converges to $T$ in some sense, and consider

$$
\tilde{x}_{\alpha, n}:=g_{\alpha}\left(T_{n}^{*} T_{n}\right) T_{n}^{*} \tilde{y}
$$

in place of $\tilde{x}_{\alpha}$. One of the well-considered finite rank approximations in the literature for the case of Tikhonov regularization is the projection method in which $T_{n}$ is taken as either $T P_{n}$ or $P_{\ell} T P_{m}$, where for each $n \in \mathbb{N}, P_{n}: X \rightarrow X$ is an orthogonal projection onto a finite-dimensional subspace $X_{n}$ of $X$.

In [4], Periverzev considered Tikhonov regularization, with

$$
T_{n}=P_{1} T P_{2^{2 n}}+\sum_{k=1}^{n}\left(P_{2^{k}}-P_{2^{k-1}}\right) T P_{2^{2 n-k}}
$$

with $R\left(P_{2^{k+1}}\right) \subseteq R\left(P_{2^{k+1}}\right)$ and showed that the computational complexity for obtaining the solution

$$
\tilde{x}_{\alpha, n}:=\left(T_{n}^{*} T_{n}+\alpha I\right)^{-1} T_{n}^{*} \tilde{y}
$$

is far less than that for ordinary projection method when $T$ and $T^{*}$ are having certain smoothness properties and $\left(P_{n}\right)$ is having certain approximation properties. 
Recently, Solodkiǔ [6] applied the above modified projection approximation to the general regularization method, and obtained error estimate for the approximation

$$
\tilde{x}_{\alpha, n}=g_{\alpha}\left(T_{n}^{*} T_{n}\right) T_{n}^{*} \tilde{y}
$$

under an a priori choice of the regularization parameter $\alpha$.

In this paper we not only consider the above class of regularization methods defined by $\tilde{x}_{\alpha, n}=g_{\alpha}\left(T_{n}^{*} T_{n}\right) T_{n}^{*} \tilde{y}$ with $T_{n}$ as in (1.14), but also consider a modified form of the generalized Arcangeli's discrepancy principle

$$
\left\|T_{n} \tilde{x}_{\alpha, n}-\tilde{f}\right\|=\frac{\left(\delta+a_{n}\right)^{p}}{\alpha^{q}}, \quad p>0, q>0,
$$

for choosing the regularization parameter $\alpha$. Here $\left(a_{n}\right)$ is a sequence of positive real numbers such that $a_{n} \rightarrow 0$ as $n \rightarrow \infty$. It is to be mentioned that, in [3], the authors considered the above discrepancy principle for Tikhonov regularization with $T_{n}$ as in (1.14). The advantage of having a general sequence $\left(a_{n}\right)$ instead of the traditional $\left(\epsilon_{n}\right)$, where $\left\|T-T_{n}\right\|=O\left(\epsilon_{n}\right)$, is that the order of convergence of the approximation is in terms of powers of $\delta+a_{n}$, in place of powers of $\delta+\epsilon_{n}$ with $a_{n}$ smaller than $\epsilon_{n}$. By properly choosing $\left(a_{n}\right)$, it can happen that, for a small $\delta$, the values of $n$ for which $a_{n}=O(\delta)$, can be much smaller than that required for $\epsilon_{n}=O(\delta)$. In this paper we are going to use the estimate $\left\|T-T_{n}\right\|=$ $O\left(\epsilon_{n}\right), \epsilon_{n}=2^{-n r}$, proved in [3], where $r>0$ is a quantity depending on the smoothness property of $T$, and take $\left(a_{n}\right)$ such that $2^{-n r}=O\left(a_{n}^{\lambda}\right)$ for some $\lambda>0$. For instance one may take $a_{n}=2^{-n r / \lambda}$ for any $\lambda \in(0,1]$.

In order to specify the smoothness properties of the operator $T$ and approximation property of $\left(P_{n}\right)$, we adopt the following setting as in [3, 4].

For $r>0$, let $X_{r}$ be a dense subspace of the Hilbert space $X$ and $L_{r}: X_{r} \rightarrow X$ a closed linear operator. On $X_{r}$ consider the inner product

$$
\langle f, g\rangle_{r}:=\langle f, g\rangle+\left\langle L_{r} f, L_{r} g\right\rangle, \quad f, g \in X_{r},
$$

and the corresponding norm

$$
\|f\|_{r}:=\|f\|+\left\|L_{r} f\right\|, \quad f \in X_{r} .
$$

It can be seen that, with respect to the above inner product $\langle\cdot, \cdot\rangle_{r}, X_{r}$ is a Hilbert space.

If $A: X \rightarrow X, B: X_{r} \rightarrow X, C: X \rightarrow X_{r}$ are bounded operators, then we will denote their norms by

$$
\|A\|, \quad\|B\|_{r, 0}, \quad\|C\|_{0, r},
$$

respectively. 
We assume that $T: X \rightarrow X$ is a compact operator having the smoothness properties

$$
R(T) \subseteq X_{r}, \quad R\left(T^{*}\right) \subseteq X_{r}, \quad R\left(\left(L_{r} T\right)^{*}\right) \subseteq X_{r}
$$

with

$$
T: X \longrightarrow X_{r}, \quad T^{*}: X \longrightarrow X_{r}, \quad\left(L_{r} T\right)^{*}: X \longrightarrow X_{r}
$$

being bounded operators, so that there exist positive real numbers $\gamma_{1}, \gamma_{2}, \gamma_{3}$ such that

$$
\|T\|_{0, r} \leq \gamma_{1}, \quad\left\|T^{*}\right\|_{0, r} \leq \gamma_{2}, \quad\left\|\left(L_{r} T\right)^{*}\right\|_{0, r} \leq \gamma_{3} .
$$

Further, we assume that $\left(P_{n}\right)$ is a sequence of orthogonal projections having the approximation property

$$
\left\|I-P_{n}\right\|_{r, 0} \leq c_{r} n^{-r}
$$

where $c_{r}>0$ is independent of $n$.

\section{Error estimate and discrepancy principle}

2.1. Error estimate. Let $T: X \rightarrow X$ be a compact operator having the smoothness properties specified by (1.21) and (1.23) and $\left(P_{n}\right)$ a sequence of orthogonal projections having the approximation property (1.24). For each $n \in \mathbb{N}$, let $T_{n}$ be defined by (1.14).

Let $y \in R(T)$ and $\tilde{y} \in X$ be such that

$$
\|y-\tilde{y}\| \leq \delta
$$

Let $\left\{g_{\alpha}: \alpha>0\right\}$ be a set of Borel measurable functions defined on $(0, b]$, where

$$
b \geq \max \left\{\|T\|^{2},\left\|T_{n}\right\|^{2}\right\} \quad \forall n \in \mathbb{N},
$$

and satisfying Assumptions 1, 2, 3, and 4. Let

$$
\begin{gathered}
\hat{x}:=T^{\dagger} y, \quad x_{\alpha}:=g_{\alpha}\left(T^{*} T\right) T^{*} y, \\
x_{\alpha, n}:=g_{\alpha}\left(T_{n}^{*} T_{n}\right) T_{n}^{*} y, \quad \tilde{x}_{\alpha, n}:=g_{\alpha}\left(T_{n}^{*} T_{n}\right) T_{n}^{*} \tilde{y} .
\end{gathered}
$$

Further we assume that $\hat{x} \in R\left(\left(T^{*} T\right)^{v}\right)$ for some $v \in\left(0, v_{0}\right]$, and

$$
\hat{x}=\left(T^{*} T\right)^{v} \hat{u}, \quad \hat{u} \in X .
$$

In order to find an estimate for the error $\left\|\hat{x}-\tilde{x}_{\alpha, n}\right\|$, first we observe that

$$
\left\|\hat{x}-\tilde{x}_{\alpha, n}\right\| \leq\left\|\hat{x}-x_{\alpha, n}\right\|+\left\|x_{\alpha, n}-\tilde{x}_{\alpha, n}\right\| .
$$


344 Arcangeli's type discrepancy principles

By the definition of $x_{\alpha, n}, \tilde{x}_{\alpha, n}$, and using spectral results, we have

$$
x_{\alpha, n}-\tilde{x}_{\alpha, n}=g_{\alpha}\left(T_{n}^{*} T_{n}\right) T_{n}^{*}(y-\tilde{y})=T_{n}^{*} g_{\alpha}\left(T_{n} T_{n}^{*}\right)(y-\tilde{y}) .
$$

Therefore, using Assumption 2 on $g_{\alpha}$, we get

$$
\begin{aligned}
\left\|x_{\alpha, n}-\tilde{x}_{\alpha, n}\right\| & =\left\|T_{n}^{*} g_{\alpha}\left(T_{n} T_{n}^{*}\right)(y-\tilde{y})\right\| \\
& =\left\|\left(T_{n} T_{n}^{*}\right)^{1 / 2} g_{\alpha}\left(T_{n} T_{n}^{*}\right)(y-\tilde{y})\right\| \\
& \leq \sup _{0 \leq \lambda \leq b} \lambda^{1 / 2}\left|g_{\alpha}(\lambda)\right|\|y-\tilde{y}\| \leq d \frac{\delta}{\sqrt{\alpha}} .
\end{aligned}
$$

Thus, we have

$$
\left\|\hat{x}-\tilde{x}_{\alpha, n}\right\| \leq\left\|\hat{x}-x_{\alpha, n}\right\|+d \frac{\delta}{\sqrt{\alpha}} .
$$

The following theorem supplies an estimate for $\left\|\hat{x}-x_{\alpha, n}\right\|$. For its proof we will make use of the result

$$
\left\|A^{\ell}-A_{n}^{\ell}\right\| \leq a_{\ell}\left\|A-A_{n}\right\|^{\min \{1, \ell\}}, \quad \ell>0,
$$

proved in [7] for positive, selfadjoint, bounded operators $A$ and $A_{n}$ on $X$, with $\left(A_{n}\right)$ uniformly bounded, where $a_{\ell}>0$ is independent of $n$.

Proposition 2.1. Let $\hat{x}$ and $x_{\alpha, n}$ be as in (2.3). Then

$$
\left\|\hat{x}-x_{\alpha, n}\right\| \leq c\left(\alpha^{\nu}+\left\|T^{*} T-T_{n}^{*} T_{n}\right\|^{\min \{1, \nu\}}+\alpha^{-1 / 2}\left\|\left(T_{n}-P_{2^{n}} T\right)\left(T^{*} T\right)^{v}\right\|\right) .
$$

Proof. We observe that

$$
\begin{aligned}
\hat{x}-x_{\alpha, n} & =\hat{x}-g_{\alpha}\left(T_{n}^{*} T_{n}\right) T_{n}^{*} T \hat{x} \\
& =\left[I-g_{\alpha}\left(T_{n}^{*} T_{n}\right) T_{n}^{*} T_{n}\right] \hat{x}+g_{\alpha}\left(T_{n}^{*} T_{n}\right) T_{n}^{*}\left(T-T_{n}\right) \hat{x},
\end{aligned}
$$

so that

$$
\left\|\hat{x}-x_{\alpha, n}\right\| \leq\left\|\left[I-g_{\alpha}\left(T_{n}^{*} T_{n}\right) T_{n}^{*} T_{n}\right] \hat{x}\right\|+\left\|g_{\alpha}\left(T_{n}^{*} T_{n}\right) T_{n}^{*}\left(T-T_{n}\right) \hat{x}\right\| .
$$

Since $\hat{x}=\left(T^{*} T\right)^{v} \hat{u}$,

$$
\begin{aligned}
\left\|\left[I-g_{\alpha}\left(T_{n}^{*} T_{n}\right) T_{n}^{*} T_{n}\right] \hat{x}\right\|= & \left\|\left[I-T_{n}^{*} T_{n} g_{\alpha}\left(T_{n}^{*} T_{n}\right)\right]\left(T^{*} T\right)^{v} \hat{u}\right\| \\
\leq & \left\|\left[I-T_{n}^{*} T_{n} g_{\alpha}\left(T_{n}^{*} T_{n}\right)\right]\left[\left(T^{*} T\right)^{v}-\left(T_{n}^{*} T_{n}\right)^{\nu}\right] \hat{u}\right\| \\
& +\left\|\left[I-T_{n}^{*} T_{n} g_{\alpha}\left(T_{n}^{*} T_{n}\right)\right]\left(T_{n}^{*} T_{n}\right)^{v} \hat{u}\right\| .
\end{aligned}
$$


Now, using Assumption 1 on $g_{\alpha}$,

$$
\left\|\left[I-T_{n}^{*} T_{n} g_{\alpha}\left(T_{n}^{*} T_{n}\right)\right]\left(T_{n}^{*} T_{n}\right)^{v} \hat{u}\right\| \leq \sup _{0<\leq \lambda \leq b} \lambda^{v}\left|1-\lambda g_{\alpha}(\lambda)\right|\|\hat{u}\| \leq c_{v}\|\hat{u}\| \alpha^{v},
$$

and by Assumption 1 on $g_{\alpha}$ and the result (2.9) with $A=T^{*} T, A_{n}=T_{n}^{*} T_{n}$ and $\ell=v$,

$$
\begin{aligned}
\left\|r_{\alpha}\left(T_{n}^{*} T_{n}\right)\left[\left(T^{*} T\right)^{\nu}-\left(T_{n}^{*} T_{n}\right)^{\nu}\right] \hat{u}\right\| & \leq\left\|r_{\alpha}\left(T_{n}^{*} T_{n}\right)\right\|\left\|\left[\left(T^{*} T\right)^{\nu}-\left(T_{n}^{*} T_{n}\right)^{\nu}\right]\right\|\|\hat{u}\| \\
& \leq c_{0}\|\hat{u}\|\left\|\left[\left(T^{*} T\right)^{v}-\left(T_{n}^{*} T_{n}\right)^{\nu}\right]\right\| \\
& \leq c_{0} a_{\nu}\|\hat{u}\|\left\|T^{*} T-T_{n}^{*} T_{n}\right\|^{\min \{1, v\}} .
\end{aligned}
$$

Since $T_{n}^{*} P_{2^{n}}=T_{n}^{*}, \hat{x}=\left(T^{*} T\right)^{v} \hat{u}$ and using Assumption 2 on $g_{\alpha}$, we have

$$
\begin{aligned}
\left\|g_{\alpha}\left(T_{n}^{*} T_{n}\right) T_{n}^{*}\left(T_{n}-T\right) \hat{x}\right\| & =\left\|g_{\alpha}\left(T_{n}^{*} T_{n}\right) T_{n}^{*}\left(T_{n}-P_{2^{n}} T\right) \hat{x}\right\| \\
& =\left\|\left(T_{n} T_{n}^{*}\right)^{1 / 2} g_{\alpha}\left(T_{n} T_{n}^{*}\right)\left(T_{n}-P_{2^{n}} T\right) \hat{x}\right\| \\
& \leq\left\|\left(T_{n} T_{n}^{*}\right)^{1 / 2} g_{\alpha}\left(T_{n} T_{n}^{*}\right)\right\|\left\|\left(T_{n}-P_{2^{n}} T\right)\left(T^{*} T\right)^{v} \hat{u}\right\| \\
& \leq d\|\hat{u}\| \alpha^{-1 / 2}\left\|\left(T_{n}-P_{2^{n}} T\right)\left(T^{*} T\right)^{v}\right\| .
\end{aligned}
$$

Using the above estimates for $\left\|\left[I-g_{\alpha}\left(T_{n}^{*} T_{n}\right) T_{n}^{*} T_{n}\right] \hat{x}\right\|$ and $\| g_{\alpha}\left(T_{n}^{*} T_{n}\right) T_{n}^{*}(T-$ $\left.T_{n}\right) \hat{x} \|$ in relation (2.12) we get the required result.

In view of relation (2.8) and Proposition 2.1, we have to find estimates for the quantities

$$
\left\|T^{*} T-T_{n}^{*} T_{n}\right\|, \quad\left\|\left(T_{n}-P_{2^{n}} T\right)\left(T^{*} T\right)^{v}\right\| .
$$

It is proved in [4] (also see [6]) that

$$
\left\|T^{*} T-T_{n}^{*} T_{n}\right\|=O\left(2^{-2 n r}\right)
$$

so that

$$
\left\|T^{*} T-T_{n}^{*} T_{n}\right\|^{\min \{1, v\}}=O\left(2^{-2 n r v_{1}}\right), \quad v_{1}=\min \{v, 1\} .
$$

Also, the estimate for $\left\|\left(T_{n}-P_{2^{n}} T\right)\left(T^{*} T\right)^{v}\right\|$ given in the following lemma can be deduced from a result of Solodkii [6]. Here we will give an independent and detailed proof for the same. We will use the estimates

$$
\left\|T\left(I-P_{m}\right)\right\|=O\left(m^{-r}\right), \quad\left\|T\left(I-P_{m}\right)\right\|_{0, r}=O\left(m^{-r}\right)
$$

obtained by Pereverzev [4] (cf. also [3]) and the estimate

$$
\left\|\left(I-P_{m}\right)|T|^{\ell}\right\|=O\left(\left\|T\left(I-P_{m}\right)\right\|^{\min \{\ell, 1\}}\right), \quad \ell>0
$$

given in [5]. 
346 Arcangeli's type discrepancy principles

Lemma 2.2. For $v>0$,

$$
\left\|\left(T_{n}-P_{2^{n}} T\right)\left(T^{*} T\right)^{v}\right\|=O\left(2^{-n r\left(2+v_{2}\right)}\right), \quad v_{2}=\min \{2 v, 1\} .
$$

Proof. It can be seen that

$$
P_{2^{n}} T-T_{n}=P_{1} T\left(I-P_{2^{2 n}}\right)+\sum_{k=1}^{n}\left(P_{2^{k}}-P_{2^{k-1}}\right) T\left(I-P_{2^{2 n-k}}\right) .
$$

Therefore,

$$
\begin{aligned}
&\left\|\left(P_{2^{n}} T-T_{n}\right)\left(T^{*} T\right)^{v}\right\| \\
& \leq\left\|T\left(I-P_{2^{2 n}}\right)\left(T^{*} T\right)^{v}\right\|+\sum_{k=1}^{n}\left\|\left(I-P_{2^{k-1}}\right) T\left(I-P_{2^{2 n-k}}\right)\left(T^{*} T\right)^{v}\right\| \\
& \leq\left\|T\left(I-P_{2^{2 n}}\right)\right\|\left\|\left(I-P_{2^{2 n}}\right)\left(T^{*} T\right)^{v}\right\| \\
&+\sum_{k=1}^{n}\left\|\left(I-P_{2^{k-1}}\right) T\left(I-P_{2^{2 n-k}}\right)\right\|\left\|\left(I-P_{2^{2 n-k}}\right)\left(T^{*} T\right)^{v}\right\| \\
& \leq\left\|T\left(I-P_{2^{2 n}}\right)\right\|\left\|\left(I-P_{2^{2 n}}\right)\left(T^{*} T\right)^{v}\right\| \\
&+\sum_{k=1}^{n}\left\|\left(I-P_{2^{k-1}}\right)\right\|_{r, 0}\left\|T\left(I-P_{2^{2 n-k}}\right)\right\|_{0, r}\left\|\left(I-P_{2^{2 n-k}}\right)\left(T^{*} T\right)^{v}\right\| .
\end{aligned}
$$

Now using (1.24), (2.20), and (2.21), it follows that

$$
\begin{aligned}
& \left\|\left(P_{2^{n}} T-T_{n}\right)\left(T^{*} T\right)^{v}\right\| \\
& \quad \leq \kappa_{1} 2^{-2 n r}\left(2^{-2 n r}\right)^{\min \{2 v, 1\}}+\kappa_{2} \sum_{k=1}^{n} 2^{-(k-1) r} 2^{-(2 n-k) r}\left[2^{-(2 n-k) r}\right]^{\min \{2 v, 1\}} \\
& \quad \leq \kappa 2^{-2 n r} 2^{-2 n r \nu_{2}} \sum_{k=0}^{n} 2^{k r \nu_{2}}, \quad v_{2}=\min \{2 v, 1\} \\
& \quad=O\left(2^{-n r\left(2+\nu_{2}\right)}\right) .
\end{aligned}
$$

Thus the lemma is proved.

Now, the estimates in (2.19) and (2.22) together with Proposition 2.1 and relation (2.8) gives the following result.

Theorem 2.3. Suppose that $\hat{x} \in R\left(\left(T^{*} T\right)^{v}\right)$ and $y \in R(T)$. Then

$$
\left\|\hat{x}-\tilde{x}_{\alpha, n}\right\| \leq c\left(\alpha^{\nu}+2^{-2 n r v_{1}}+\frac{2^{-n r\left(2+\nu_{2}\right)}}{\sqrt{\alpha}}+\frac{\delta}{\sqrt{\alpha}}\right),
$$


where

$$
v_{1}=\min \{v, 1\}, \quad v_{2}=\min \{2 v, 1\} .
$$

2.2. Discrepancy principle. We consider the discrepancy principle

$$
\left\|T_{n} \tilde{x}_{\alpha, n}-\tilde{y}\right\|=\frac{\left(\delta+a_{n}\right)^{p}}{\alpha^{q}}, \quad p>0, q>0,
$$

where $\left(a_{n}\right)$ is a sequence of positive reals such that $a_{n} \rightarrow 0$ as $n \rightarrow 0$.

Let

$$
f_{n}(\alpha, \tilde{y})=\alpha^{q}\left\|T_{n} \tilde{x}_{\alpha, n}-\tilde{y}\right\| .
$$

We observe that

$$
T_{n} \tilde{x}_{\alpha, n}-\tilde{y}=\left[T_{n} T_{n}^{*} g_{\alpha}\left(T_{n} T_{n}^{*}\right)-I\right] \tilde{y} .
$$

Hence, by Assumptions 1 and 3 on $g_{\alpha}, \alpha>0$, and using spectral theory, we have

$$
\begin{aligned}
\left\|T_{n} \tilde{x}_{\alpha, n}-\tilde{y}\right\| & =\left\|\left[T_{n} T_{n}^{*} g_{\alpha}\left(T_{n} T_{n}^{*}\right)-I\right] \tilde{y}\right\| \leq \sup _{0<\lambda \leq b}\left|1-\lambda g_{\alpha}(\lambda)\right|\|\tilde{y}\| \leq c_{0}, \\
\left\|T_{n} \tilde{x}_{\alpha, n}-\tilde{y}\right\|^{2} & =\left\|\left[T_{n} T_{n}^{*} g_{\alpha}\left(T_{n} T_{n}^{*}\right)-I\right] \tilde{y}\right\|^{2}=\int_{0}^{b}\left[1-\lambda g_{\alpha}(\lambda)\right]^{2} d\left\|E_{\lambda} \tilde{y}\right\|^{2} \\
& \geq \int_{0}^{b}\left(\kappa_{0} \alpha^{\nu_{0}}\right)^{2} d\left\|E_{\lambda} \tilde{y}\right\|^{2} \geq\left(\kappa_{0} \alpha^{\nu_{0}}\|\tilde{y}\|\right)^{2} .
\end{aligned}
$$

Therefore, it follows that

$$
\lim _{\alpha \rightarrow 0} f_{n}(\alpha, \tilde{y})=0, \quad \lim _{\alpha \rightarrow \infty} f_{n}(\alpha, \tilde{y})=\infty
$$

Hence by the intermediate value theorem and Assumption 4 on $\left\{g_{\alpha}\right\}$, there exists a unique $\alpha$ satisfying the discrepancy principle (2.28). It also follows that

$$
\frac{\left(\delta+a_{n}\right)^{p}}{\alpha^{q}}=\left\|T_{n} \tilde{x}_{\alpha, n}-\tilde{y}\right\| \geq \kappa_{0} \alpha^{\nu_{0}}\|\tilde{y}\|
$$

so that

$$
\alpha=O\left(\delta+a_{n}\right)^{p /\left(q+v_{0}\right)} .
$$

For the next result we make use of the estimate

$$
\left\|T-T_{n}\right\|=O\left(2^{-n r}\right)
$$

proved in [3]. 
Proposition 2.4. Suppose that $\hat{x} \in R\left(T^{*} T\right)^{v}$ for some $v$ with $0<v \leq v_{0},\left(a_{n}\right)$ is such that $2^{-n r}=O\left(a_{n}^{\lambda}\right)$ for some $\lambda>0$ and $\alpha$ is chosen according to the discrepancy principle (2.28). Then

$$
\frac{\left(\delta+a_{n}\right)^{p}}{\alpha^{q}}=O\left(\left(\delta+a_{n}\right)^{s}\right),
$$

where

$$
\begin{aligned}
& s=\min \left\{1, \lambda, \frac{p \omega}{q+v_{0}}, \frac{p}{2\left(q+v_{0}\right)}+2 \lambda v_{2}\right\}, \\
& \nu_{2}=\min \{v, 1\}, \quad \omega=\min \left\{v+\frac{1}{2}, v_{0}\right\} .
\end{aligned}
$$

Proof. From the discrepancy principle (2.28) we have

$$
\begin{aligned}
\frac{\left(\delta+a_{n}\right)^{p}}{\alpha^{q}} & =\left\|T_{n} \tilde{x}_{\alpha, n}-\tilde{y}\right\|=\left\|\left[I-g_{\alpha}\left(T_{n} T_{n}^{*}\right) T_{n} T_{n}^{*}\right] \tilde{y}\right\| \\
& =\left\|\left[I-g_{\alpha}\left(T_{n} T_{n}^{*}\right) T_{n} T_{n}^{*}\right] y\right\|+\left\|\left[I-g_{\alpha}\left(T_{n} T_{n}^{*}\right) T_{n} T_{n}^{*}\right](\tilde{y}-y)\right\| .
\end{aligned}
$$

We observe that

$$
\begin{aligned}
\left\|\left[I-g_{\alpha}\left(T_{n} T_{n}^{*}\right) T_{n} T_{n}^{*}\right] y\right\|= & \left\|\left[I-g_{\alpha}\left(T_{n} T_{n}^{*}\right) T_{n} T_{n}^{*}\right]\left(T-T_{n}\right) \hat{x}\right\| \\
& +\left\|\left[I-g_{\alpha}\left(T_{n} T_{n}^{*}\right) T_{n} T_{n}^{*}\right] T_{n} \hat{x}\right\| \\
= & \left\|\left[I-T_{n} T_{n}^{*} g_{\alpha}\left(T_{n} T_{n}^{*}\right)\right]\left(T-T_{n}\right) \hat{x}\right\| \\
& +\left\|\left[I-T_{n} T_{n}^{*} g_{\alpha}\left(T_{n} T_{n}^{*}\right)\right] T_{n} \hat{x}\right\| .
\end{aligned}
$$

Now, using the fact that $\hat{x}=\left(T^{*} T\right)^{v} \hat{u}$, Assumption 1 on $g_{\alpha}, \alpha>0$, and spectral results, we have

$$
\begin{aligned}
\left\|\left[I-T_{n} T_{n}^{*} g_{\alpha}\left(T_{n} T_{n}^{*}\right)\right] T_{n} \hat{x}\right\|= & \left\|\left(T_{n}^{*} T_{n}\right)^{1 / 2}\left[I-T_{n}^{*} T_{n} g_{\alpha}\left(T_{n}^{*} T_{n}\right)\right]\left(T^{*} T\right)^{v} \hat{u}\right\| \\
= & \left\|\left(T_{n}^{*} T_{n}\right)^{1 / 2}\left[I-T_{n}^{*} T_{n} g_{\alpha}\left(T_{n}^{*} T_{n}\right)\right]\left(T_{n}^{*} T_{n}\right)^{v} \hat{u}\right\| \\
& +\|\left(T_{n}^{*} T_{n}\right)^{1 / 2}\left[I-T_{n}^{*} T_{n} g_{\alpha}\left(T_{n}^{*} T_{n}\right)\right] \\
& \times\left[\left(T^{*} T\right)^{v}-\left(T_{n}^{*} T_{n}\right)^{\nu}\right] \hat{u} \| \\
\leq & \hat{c}_{\nu} \alpha^{\omega}\|\hat{u}\|+c_{1 / 2} \alpha^{1 / 2}\|\hat{u}\|\left\|\left(T^{*} T\right)^{v}-\left(T_{n}^{*} T_{n}\right)^{v}\right\|,
\end{aligned}
$$

where $\hat{c}_{v}=c_{v+1 / 2}$ if $v+1 / 2 \leq v_{0}$ and $\hat{c}_{v}=c_{\nu_{0}}$ if $v+1 / 2 \geq v_{0}$, and $\omega=$ $\min \left\{v+1 / 2, v_{0}\right\}$. Hence

$$
\begin{aligned}
\left\|\left[I-g_{\alpha}\left(T_{n} T_{n}^{*}\right) T_{n} T_{n}^{*}\right] y\right\| \leq & c_{0}\left\|\left(T-T_{n}\right) \hat{x}\right\|+c_{\nu} \alpha^{\omega}\|\hat{u}\| \\
& +c_{1 / 2} \alpha^{1 / 2}\|\hat{u}\|\left\|\left(T^{*} T\right)^{\nu}-\left(T_{n}^{*} T_{n}\right)^{\nu}\right\| .
\end{aligned}
$$


Also, we have

$$
\left\|\left(I-g_{\alpha}\left(T_{n} T_{n}^{*}\right)\right) T_{n} T_{n}^{*}(\tilde{y}-y)\right\| \leq c_{0} \delta
$$

Thus

$$
\begin{aligned}
\frac{\left(\delta+a_{n}\right)^{p}}{\alpha^{q}} \leq & c_{0}\left\|\left(T-T_{n}\right) \hat{x}\right\|+c_{\nu} \alpha^{\omega}\|\hat{u}\|+c_{1 / 2} \alpha^{1 / 2}\|\hat{u}\| \\
& \times\left\|\left(T^{*} T\right)^{v}-\left(T_{n}^{*} T_{n}\right)^{v}\right\|+c_{0} \delta
\end{aligned}
$$

Now by the results $(2.9),(2.34),(2.35)$, and the assumption that $2^{-n r}=O\left(a_{n}^{\lambda}\right)$, we have

$$
\begin{aligned}
\frac{\left(\delta+a_{n}\right)^{p}}{\alpha^{q}} \leq & c\left(a_{n}^{\lambda}+\alpha^{\omega}+\alpha^{1 / 2} a_{n}^{2 \lambda v_{2}}+\delta\right) \\
\leq & c\left(\left(\delta+a_{n}\right)^{\lambda}+\alpha^{\omega}+\alpha^{1 / 2}\left(\delta+a_{n}\right)^{2 \lambda \nu_{2}}+\left(\delta+a_{n}\right)\right) \\
\leq & c\left(\left(\delta+a_{n}\right)^{\lambda}+\left(\delta+a_{n}\right)^{p \omega /\left(q+v_{o}\right)}\right. \\
& \left.\quad+\left(\delta+a_{n}\right)^{\left(p / 2\left(q+v_{0}\right)\right)+2 \lambda v_{2}}+\left(\delta+a_{n}\right)\right)
\end{aligned}
$$

where $v_{2}=\min \{v, 1\}, \omega=\min \left\{v+1 / 2, v_{o}\right\}$. Thus

$$
\begin{gathered}
\frac{\left(\delta+a_{n}\right)^{p}}{\alpha^{q}}=O\left(\left(\delta+a_{n}\right)^{s}\right), \\
s=\min \left\{1, \lambda, \frac{p}{2\left(q+v_{0}\right)}+2 \lambda v_{2}, \frac{p \omega}{q+v_{0}}\right\} .
\end{gathered}
$$

THEOREM 2.5. In addition to the assumptions in Proposition 2.4, suppose that

$$
p<s+2 q \min \left\{1, \lambda\left(2+v_{2}\right)\right\}
$$

where

$$
\begin{gathered}
s=\min \left\{1, \lambda, \frac{p \omega}{q+v_{0}}, \frac{p}{2\left(q+v_{0}\right)}+2 \lambda v_{2}\right\}, \\
\omega=\min \left\{v+\frac{1}{2}, v_{0}\right\}, \quad v_{1}=\min \{v, 1\}, \quad v_{2}=\min \{2 v, 1\} .
\end{gathered}
$$

Then

$$
\begin{gathered}
\mu:=\min \left\{\frac{p v}{q+v_{0}}, 1-\frac{p}{2 q}+\frac{s}{2 q}, \lambda\left(2+v_{2}\right)-\frac{p}{2 q}+\frac{s}{2 q}\right\}>0, \\
\left\|\hat{x}-\tilde{x}_{\alpha, n}\right\|=O\left(\left(\delta+a_{n}\right)^{\mu}\right) .
\end{gathered}
$$


Proof. Clearly, $p \leq s+2 q \min \left\{1, \lambda\left(2+v_{2}\right)\right\}$ implies $\mu>0$. Now to obtain the estimate for $\left\|\hat{x}-\tilde{x}_{\alpha, n}\right\|$, first we recall from Theorem 2.3 that

$$
\left\|\hat{x}-\tilde{x}_{\alpha, n}\right\| \leq c\left(\alpha^{v}+2^{-2 n r v_{1}}+\frac{2^{-n r\left(2+v_{2}\right)}}{\sqrt{\alpha}}+\frac{\delta}{\sqrt{\alpha}}\right) .
$$

Now, using the assumption that $2^{-n r}=O\left(a_{n}^{\lambda}\right)$ for some $\lambda>0$, and relation (2.34), we have

$$
\begin{aligned}
\left\|\hat{x}-\tilde{x}_{\alpha, n}\right\| & \leq c\left(\left(\delta+a_{n}\right)^{p v /\left(q+v_{0}\right)}+a_{n}^{2 \lambda v_{1}}+\frac{a_{n}^{\lambda\left(2+v_{2}\right)}}{\sqrt{\alpha}}+\frac{\delta}{\sqrt{\alpha}}\right) \\
& \leq c\left(\left(\delta+a_{n}\right)^{p v /\left(q+v_{0}\right)}+\left(\delta+a_{n}\right)^{2 \lambda v_{1}}+\frac{\left(\delta+a_{n}\right)^{\lambda\left(2+v_{2}\right)}}{\sqrt{\alpha}}+\frac{\delta+a_{n}}{\sqrt{\alpha}}\right) .
\end{aligned}
$$

Since

$$
\frac{\left(\delta+a_{n}\right)^{\ell}}{\sqrt{\alpha}}=\left(\delta+a_{n}\right)^{\ell-p / 2 q}\left[\frac{\left(\delta+a_{n}\right)^{p}}{\alpha^{q}}\right]^{1 / 2 q}
$$

for any $\ell>0$, by Proposition 2.4,

$$
\begin{gathered}
\frac{\left(\delta+a_{n}\right)}{\sqrt{\alpha}}=O\left(\left(\delta+a_{n}\right)^{1-(p / 2 q)+(s / 2 q)}\right), \\
\frac{\left(\delta+a_{n}\right)^{\lambda\left(2+v_{2}\right)}}{\sqrt{\alpha}}=O\left(\left(\delta+a_{n}\right)^{\lambda\left(2+v_{2}\right)-(p / 2 q)+(s / 2 q)}\right) .
\end{gathered}
$$

Thus

$$
\left\|\hat{x}-\tilde{x}_{\alpha, n}\right\|=O\left(\left(\delta+a_{n}\right)^{\mu}\right)
$$

The following corollary whose proof is immediate from the above theorem, specifies a condition required to be satisfied by $\lambda$, and there by the sequence $\left(a_{n}\right)$, so as to yield a somewhat realistic error estimate.

Corollary 2.6. In addition to the assumption in Theorem 2.5, suppose $\lambda, p, q$ are such that

$$
\frac{p}{q+v_{0}} \max \left\{v_{0}, \frac{1}{2}\right\} \leq \lambda \leq 1 .
$$

Then $s$ and $\mu$ in Theorem 2.5 are given by

$$
s=\frac{p \omega}{q+v_{0}}, \quad \mu=\min \left\{\frac{p v}{q+v_{0}}, 1-\frac{p}{2\left(q+v_{0}\right)}\left(1+\frac{v_{0}-\omega}{q}\right)\right\} .
$$


In particular, with $\lambda$ as above, we have the following:

$$
\begin{array}{ll}
\mu=\frac{p v}{q+v_{0}} & \text { whenever } \frac{p}{q+v_{0}} \leq \frac{2}{2 v+1+\left(v_{0}-\omega\right) / q}, \\
\mu & =\frac{2 v}{2 v+1} \quad \text { whenever } \frac{p}{q+v_{0}}=\frac{2}{2 v+1}, v_{0}-\frac{1}{2} \leq v \leq v_{0}, \\
\mu & =\frac{2 v}{2 v_{0}+1} \quad \text { whenever } \frac{p}{q+v_{0}}=\frac{2}{2 v_{0}+1}, q \geq \frac{1}{2} .
\end{array}
$$

We may observe that the result in (2.58) of Corollary 2.6 shows that the choice of $p, q$ in the discrepancy principle (2.28) does not depend on the smoothness of the unknown solution $\hat{x}$. Also, from the above corollary we can infer that for the Arcangeli's discrepancy principle

$$
\left\|T_{n} \tilde{x}_{\alpha, n}-\tilde{y}\right\|=\frac{\delta+a_{n}}{\sqrt{\alpha}},
$$

one obtains the error estimate

$$
\left\|\hat{x}-\tilde{x}_{\alpha, n}\right\|=O\left(\left(\delta+a_{n}\right)^{\mu}\right), \quad \mu=\frac{2 v}{2 v_{0}+1},
$$

provided $\left(a_{n}\right)$ satisfies

$$
2^{-n r}=O\left(a_{n}^{\lambda}\right), \quad \max \left\{\frac{2 v_{0}}{2 \nu_{0}+1}, \frac{1}{2}\right\} \leq \lambda \leq 1 .
$$

In particular, for Tikhonov regularization, where $v_{0}=1$, we have the order $O\left(\left(\delta+a_{n}\right)^{2 v / 3}\right)$ whenever $2 / 3 \leq \lambda \leq 1$.

\section{Numerical example}

In this section, we carry out some numerical experiments using JAVA programming for Tikhonov regularization, and implement our discrepancy principle. We also implement the a priori parameter choice strategy numerically.

Consider the Hilbert space $X=Y=L^{2}[0,1]$ with the Haar orthonormal basis $\left\{e_{1}, e_{2}, \ldots,\right\}$, of piecewise constant functions, where $e_{1}(t)=1$ for all $t \in[0,1]$, and for $m=2^{k-1}+j, k=1,2, \ldots, j=1,2, \ldots, 2^{k-1}$,

$$
e_{m}(t)= \begin{cases}2^{(k-1) / 2} & \text { if } t \in\left[\frac{j-1}{2^{k-1}}, \frac{j-1 / 2}{2^{k-1}}\right), \\ -2^{(k-1) / 2} & \text { if } t \in\left[\frac{j-1 / 2}{2^{k-1}}, \frac{j}{2^{k-1}}\right), \\ 0 & \text { if } t \notin\left[\frac{j-1}{2^{k-1}}, \frac{j}{2^{k-1}}\right] .\end{cases}
$$


352 Arcangeli's type discrepancy principles

Let $T: X \rightarrow X$ be the integral operator,

$$
(T x)(s)=\int_{0}^{1} k(s, t) x(t) d t, \quad s \in[0,1],
$$

with the kernel

$$
k(s, t)= \begin{cases}t(1-s), & t \leq s, \\ s(1-t), & t>s .\end{cases}
$$

We take $X^{r}$ with $r=1$ as the Sobolev space of functions $f$ with derivative $f^{\prime} \in L^{2}[0,1]$. In all the following examples, we have $\hat{x} \in R\left(\left(T^{*} T\right)^{v}\right)$ with $2 v \leq 1$. In this case the error estimate in Theorem 2.3 takes the form

$$
\left\|\hat{x}-\tilde{x}_{\alpha, n}\right\| \leq c\left(\alpha^{v}+2^{-2 n v}+\frac{2^{-2 n(1+v)}}{\sqrt{\alpha}}+\frac{\delta}{\sqrt{\alpha}}\right) .
$$

Taking the a priori choice of the parameter $\alpha$ as

$$
\alpha \sim 2^{-2 n}, \quad \alpha \sim \delta^{2 /(2 v+1)},
$$

we get the optimal order

$$
\left\|\hat{x}-\tilde{x}_{\alpha, n}\right\|=O\left(\delta^{2 v /(2 v+1)}\right) .
$$

In a posteriori case, we find $\alpha$ using Newton-Raphson method, namely

$$
\alpha_{k+1}=\alpha_{k}-\frac{g\left(\alpha_{k}\right)}{g^{\prime}\left(\alpha_{k}\right)}, \quad k=0,1, \ldots,
$$

where

$$
\begin{aligned}
g(\alpha)= & \alpha^{2 q}\left(\bar{x}^{T} M C \bar{x}-2 \bar{x}^{T} C B+\langle\tilde{y}, \tilde{y}\rangle\right)-\left(\delta+a_{n}\right)^{2 p}, \\
g^{\prime}(\alpha)= & 2 q \alpha^{2 q-1}\left(\bar{x}^{T} M C \bar{x}-2 \bar{x}^{T} C B+\langle\tilde{y}, \tilde{y}\rangle\right) \\
& -\alpha^{2 q}\left[\bar{x}^{T} M C(\alpha+M)^{-1} \bar{x}-\bar{x}^{T}(\alpha+M)^{-1} M C \bar{x}-2 \bar{x}^{T}(\alpha+M)^{-1} C B\right],
\end{aligned}
$$

with

$$
\begin{aligned}
\bar{x} & =\left(x_{1}, x_{2}, \ldots, x_{m}\right), \\
{[B]_{i} } & =\left\langle e_{i}, \tilde{y}\right\rangle, \quad i=1,2, \ldots, m, \\
{[M]_{i j} } & =\sum_{r=1}^{2 n-v}\left(e_{i}, A e_{r}\right)\left(e_{j}, A e_{r}\right), \quad i, j=1,2, \ldots, 2^{n}, \\
{[C]_{i j} } & =\left\langle\phi_{i}, \phi_{j}\right\rangle, \quad \phi_{1}=P_{2^{2 n}} T^{*} e_{1}, \phi_{i}=P_{2^{2 n-\ell}} T^{*} e_{i}, \\
& i \in\left(2^{\ell-1}, 2^{\ell}\right], \ell=1,2, \ldots, n .
\end{aligned}
$$


Here we used the notation $[A]_{i j}$ for the $i j$ th entry of an $n \times n$ matrix $A$ and $[B]_{i}$ for the $i$ th entry of an $n \times 1$ (column) matrix $B$.

In the following examples, we take the perturbed data $\tilde{y}$ as

$$
\tilde{y}(s)=y(s)+\delta, \quad 0 \leq s \leq 1 .
$$

For the a posteriori case, we take $p$ and $q$ such that $p /(q+1)=2 / 3$, and $a_{n}=$ $\left(2^{-n}\right)^{1 / \lambda}$ with $\lambda=2 / 3$. As per Corollary 2.6, the rate is $O\left(\left(\delta+a_{n}\right)^{p v /(q+1)}\right)$. We will use the notation $\tilde{e}_{\alpha, n}$ for the computed value of $\left\|\hat{x}-\tilde{x}_{\alpha, n}\right\|$.

Example 3.1. Let $y(s)=(1 / 6)\left(s-s^{3}\right)$. In this case, it can be seen that $\hat{x}(t)=t$, $t \in[0,1]$. It is known (cf. [2]) that $\hat{x} \in R\left(T^{*} T\right)^{v}$ for all $v<1 / 8$. In the following two cases we take $v=1 / 9$.

A priori case

\begin{tabular}{rrrccl}
\hline$\delta$ & $n$ & $m$ & $\tilde{e}_{\alpha, n}$ & $\delta^{\frac{2 v}{2 v+1}}$ & $\tilde{e}_{\alpha, n} \cdot \delta^{\frac{-2 v}{2 v+1}}$ \\
\hline \multirow{3}{*}{$2^{-1.22 n}$} & 2 & 4 & 0.9059731 & 0.7371346 & 1.229047 \\
& 3 & 8 & 0.7722685 & 0.6328782 & 1.220248 \\
& 4 & 16 & 0.4068352 & 0.5433674 & 0.7487295 \\
\hline
\end{tabular}

A posteriori case

\begin{tabular}{cccrlll}
\hline$p, q$ & $\delta$ & $n$ & $m$ & $\tilde{e}_{\alpha, n}$ & $\left(\delta+a_{n}\right)^{\frac{p v}{q+1}}$ & $\tilde{e}_{\alpha, n} .\left(\delta+a_{n}\right)^{\frac{-p v}{q+1}}$ \\
\hline$p=1$ & & 2 & 4 & 0.5102194 & 0.89450734 & 0.5703915 \\
$q=1 / 2$ & $2^{-1.22 n}$ & 3 & 8 & 0.4890685 & 0.8196771 & 0.5966605 \\
& & 4 & 16 & 0.3504178 & 0.7517244 & 0.4661520 \\
\hline$p=2$ & \multirow{2}{*}{$2^{-1.22 n}$} & 2 & 4 & 0.4000930 & 0.89450734 & 0.4482135 \\
$q=2$ & & 4 & 0.3664487 & 0.8196771 & 0.4470647 \\
& & 16 & 0.3294871 & 0.7517244 & 0.43830837 \\
$p=1$ & & 4 & 0.5754841 & 0.8414794 & 0.6838956 \\
$q=1 / 2$ & $10^{-10}$ & 3 & 8 & 0.5430453 & 0.7719075 & 0.7035708 \\
& & 4 & 16 & 0.2975858 & 0.7187710 & 0.4202669 \\
\hline \multirow{2}{*}{$p=2$} & & 2 & 4 & 0.5395960 & 0.8414794 & 0.6412471 \\
$q=2$ & $10^{-10}$ & 3 & 8 & 0.4648603 & 0.7719075 & 0.6022228 \\
& & 4 & 16 & 0.28503888 & 0.7187710 & 0.3965642 \\
\hline
\end{tabular}

Example 3.2. Let $y(s)=(1 / 24)\left(s-2 s^{3}+s^{4}\right)$. In this case, $\hat{x}(t)=(1 / 2)\left(t-t^{3}\right), t \in[0,1]$ and $\hat{x} \in R\left(T^{*} T\right)^{v}$ for all $v<5 / 8$ (cf. [2]). 
354 Arcangeli's type discrepancy principles

A priori case

\begin{tabular}{ccrlll}
\hline$\delta$ & $n$ & $m$ & $\tilde{e}_{\alpha, n}$ & $\delta^{\frac{2 v}{2 v+1}}$ & $\tilde{e}_{\alpha, n} \cdot \delta^{\frac{-2 v}{2 v+1}}$ \\
\hline \multirow{3}{*}{$2^{-2 n} / 2$} & 2 & 4 & 0.2362887 & 0.1767766 & 1.3366517 \\
& 3 & 8 & 0.09444126 & 0.08838834 & 1.0681567 \\
& 4 & 16 & 0.043338350 & 0.04419417 & 0.98063492 \\
\hline
\end{tabular}

A posteriori case

\begin{tabular}{|c|c|c|c|c|c|c|}
\hline$p, q$ & $\delta$ & $n$ & $m$ & $\tilde{e}_{\alpha, n}$ & $\left(\delta+a_{n}\right)^{\frac{p v}{q+1}}$ & $\tilde{e}_{\alpha, n} \cdot\left(\delta+a_{n}\right)^{\frac{-p v}{q+1}}$ \\
\hline \multirow{3}{*}{$\begin{array}{c}p=1 \\
q=1 / 2\end{array}$} & \multirow{3}{*}{$2^{-2 * n} / 2$} & 2 & 4 & 0.08955768 & 0.54195173 & 0.16525029 \\
\hline & & 3 & 8 & 0.08927489 & 0.37696366 & 0.23682611 \\
\hline & & 4 & 16 & 0.08501988 & 0.26363660 & 0.32261129 \\
\hline \multirow{3}{*}{$\begin{array}{c}p=4 / 3 \\
q=1\end{array}$} & \multirow{3}{*}{$2^{-2 * n} / 2$} & 2 & 4 & 0.07940677 & 0.54195173 & 0.1465200 \\
\hline & & 3 & 8 & 0.0774004 & 0.37696366 & 0.2053259 \\
\hline & & 4 & 16 & 0.0683534 & 0.26363660 & 0.2593698 \\
\hline \multirow{3}{*}{$\begin{array}{c}p=1 \\
q=1 / 2\end{array}$} & \multirow{3}{*}{$10^{-10}$} & 2 & 4 & 0.09125593 & 0.50347777 & 0.18125116 \\
\hline & & 3 & 8 & 0.09081976 & 0.35724853 & 0.25422012 \\
\hline & & 4 & 16 & 0.0865327 & 0.2534898 & 0.34136562 \\
\hline \multirow{3}{*}{$\begin{array}{c}p=4 / 3 \\
q=1\end{array}$} & \multirow{3}{*}{$10^{-10}$} & 2 & 4 & 0.09045663 & 0.50347777 & 0.17966361 \\
\hline & & 3 & 8 & 0.0857890 & 0.35724853 & 0.24013831 \\
\hline & & 4 & 16 & 0.073404813 & 0.2534898 & 0.2895769 \\
\hline
\end{tabular}

\section{References}

[1] H. W. Engl, M. Hanke, and A. Neubauer, Regularization of Inverse Problems, Mathematics and its Applications, vol. 375, Kluwer, Dordrecht, 1996. MR 97k:65145. Zbl 0859.65054.

[2] H. Gfrerer, An a posteriori parameter choice for ordinary and iterated Tikhonov regularization of ill-posed problems leading to optimal convergence rates, Math. Comp. 49 (1987), no. 180, 507-522. MR 88k:65049. Zbl 0631.65056.

[3] M. T. Nair and M. P. Rajan, Arcangeli's discrepancy principle for a modified projection scheme for ill-posed problems, Numer. Funct. Anal. Optim. 22 (2001), no. 1-2, 177-198. CMP 1841868.

[4] S. V. Pereverzev, Optimization of projection methods for solving ill-posed problems, Computing 55 (1995), no. 2, 113-124. MR 96g:65062. Zbl 0830.65044.

[5] R. Plato and G. Vaŭnikko, On the regularization of projection methods for solving ill-posed problems, Numer. Math. 57 (1990), no. 1, 63-79. MR 91h:65097. Zbl 0675.65053.

[6] S. G. Solodki1̌, A modification of a projection scheme for solving ill-posed problems, Russian Math. (Iz. VUZ) 42 (1998), no. 11, 79-86, [translated from Izv. Vyssh. Uchebn. Zaved. Mat. 1998, no. 11, 83-90. MR 99m:65117]. Zbl 0961.65050. 
[7] G. M. Vaĭnikko and A. Yu. Veretennikov, Iteratsionnye protsedury v nekorrektnykh zadachakh [Iteration Procedures in Ill-Posed Problems], Nauka, Moscow, 1986 (Russian). MR 88c:47019.

M. T. Nair: Department of Mathematics, Indian Institute of Technology Madras, Chennai 600 036, India

E-mail address: mtnair@iitm.ac.in

M. P. Rajan: Department of Mathematics, Indian Institute of Technology Madras, Chennai 600 036, India 


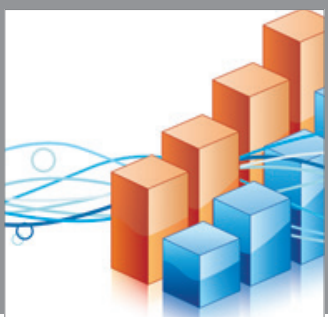

Advances in

Operations Research

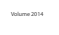

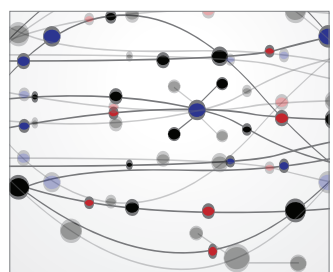

\section{The Scientific} World Journal
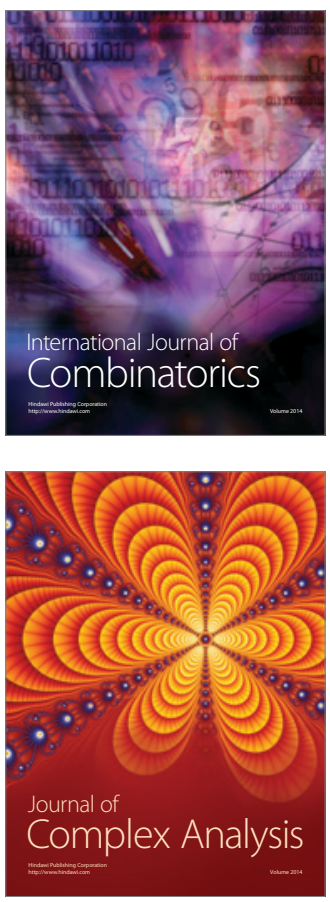

International Journal of

Mathematics and

Mathematical

Sciences
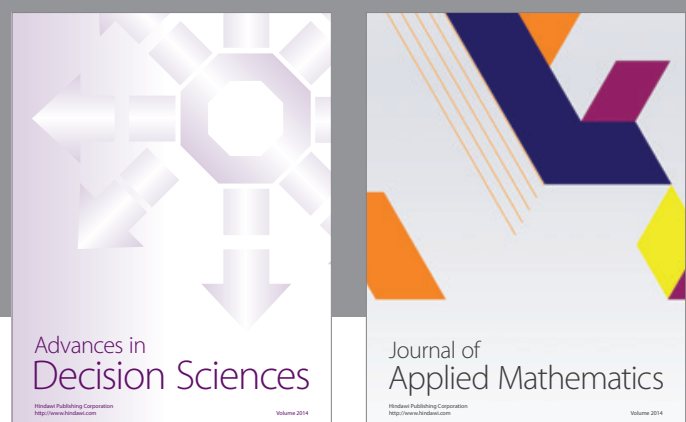

Journal of

Applied Mathematics
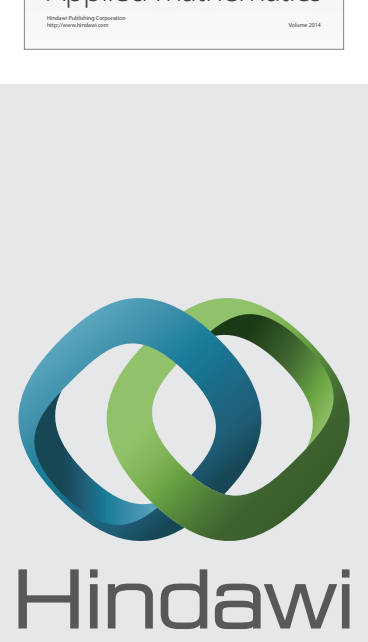

Submit your manuscripts at http://www.hindawi.com
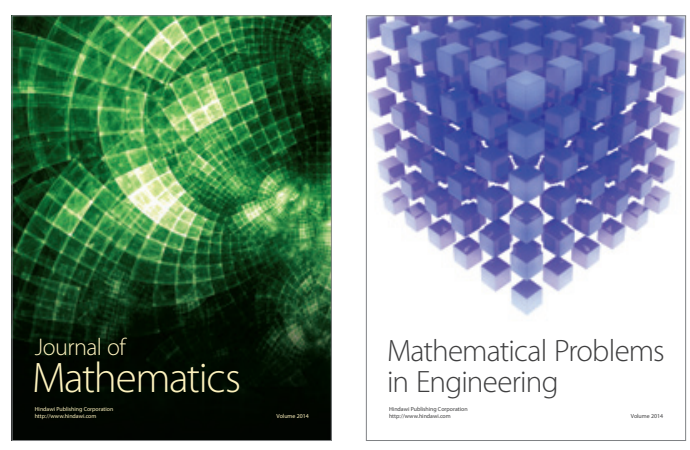

Mathematical Problems in Engineering
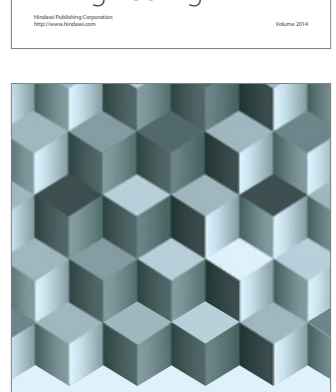

Journal of

Function Spaces
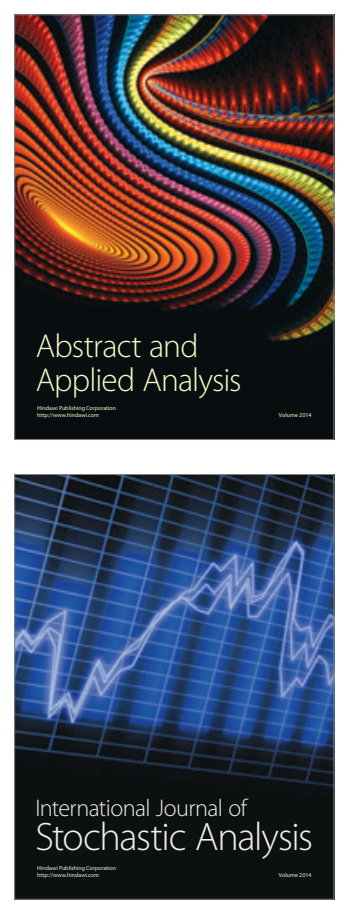

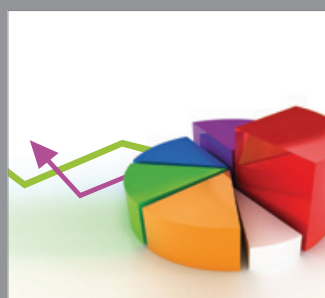

ournal of

Probability and Statistics

Promensencen
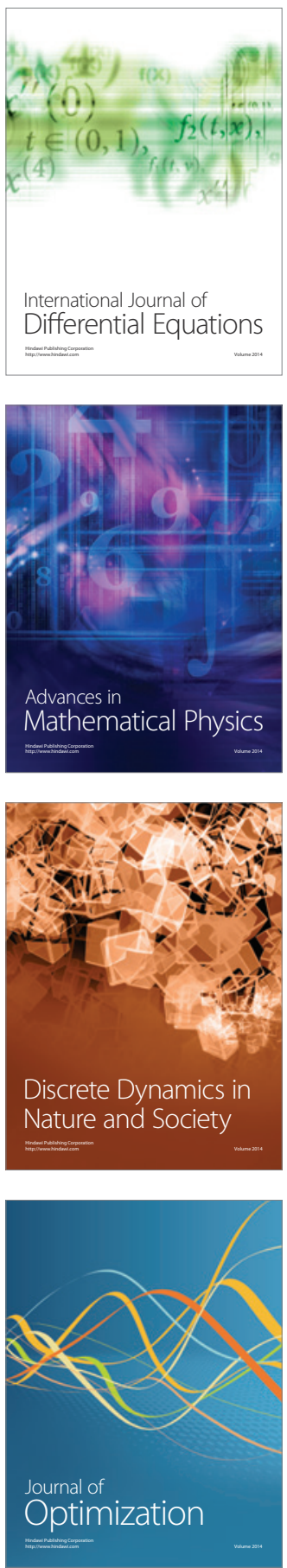